\title{
Efficacy of Anterior Gastric Fundoplication in the Treatment of Gastroesophageal Reflux in Infants and Children
}

\author{
By David Bliss, Ronald Hirschl, Keith Oldham, Janine Chey, Robert Cilley, \\ Caroline Blane, Alan Olson, Barry Shulkin, and Arnold Coran \\ Ann Arbor, Michigan
}

\begin{abstract}
- Anterior gastric fundoplication (AGF) has been performed at the University of Michigan since 1988. To objectively evaluate the long-term efficacy of the AGF, the authors performed a study of children who had undergone AGF between June 1988 and June 1990 ( $n=46$ ). Six of them died of unrelated causes. Twenty-two consented to follow-up evaluation that included parental interview, physical examination, upper gastrointestinal series (UGI), 24-hour esophageal pH probe monitoring (EpH), and a liquid-phase gastric emptying study. Twenty patients $(74 \%)$ remained asymptomatic, and only one child required reoperation. Four asymptomatic patients exhibited gastroesophageal reflux (GER) by UGI, and three others by EpH. Three children were noted to have delayed gastric emptying. These results compare favorably with data previously reported from this institution of a 5-year follow-up of children after Nissen fundoplication. There is a trend toward improved efficacy $(87 \% \vee 74 \%$; $P=.12)$, decreased reoperation rate $(4 \% \vee 14 \% ; P=.11)$, and less severe complications. The present study shows that AGF is effective treatment for GER when evaluated by objective studies and is comparable in therapeutic efficacy and safety to the Nissen fundoplication.

Copyright 11994 by W.B. Saunders Company
\end{abstract}

INDEX WORDS: Gastroesophageal reflux, fundoplication, infants and children.

$\mathbf{G}^{\mathrm{A}}$ ASTROESOPHAGEAL REFLUX (GER) is frequently diagnosed in infancy or childhood and is a known precipitating factor in a number of medical illnesses including malnutrition, aspiration, pneumonia, asthma, and esophagitis. Until recently, the most commonly performed operation for this condition in children at the C.S. Mott Children's Hospital has been the circumferential Nissen-type gastric fundoplication. As previously reported from this institution, the long-term success rate for this operation in children is nearly $75 \%$, but it is associated with a number of complications. ${ }^{1}$

As an alternative to the circumferential Nissen fundoplication (NFP), a number of centers have employed an anterior fundoplication (AGF) for the treatment of GER in the pediatric population. This approach has the practical advantages of technical simplicity and reduced operative time. The medical benefits include a reduced likelihood of distal esophageal narrowing or obstruction and the preservation of the ability to eructate or vomit under physiological conditions. In an attempt to objectively evaluate the AGF and to compare its long-term efficacy with that of the NFP, a study of patients who had undergone the former procedure was performed.

\section{MATERIALS AND METHODS}

Between 1988 and 1990, 46 patients underwent AGF for GER at the C.S. Mott Children's Hospital. The patients' records were reviewed, and attempts were made to contact all families. Twentytwo families $(53 \%)$ consented to participation in the study according to the guidelines set by the Institutional Review Board. Six children had died of unrelated illnesses before the study was begun.

Indications for operation were uniform and included appropriate symptomatology and objective findings consistent with GER, noted by an upper gastrointestinal series (UGI) or by esophageal $\mathrm{pH}$ probe monitoring (EpH).

All initial fundoplication operations were performed using a standardized technique, as shown in Fig 1. After circumferential mobilization of the distal esophagus, the posterior crural defect was closed primarily. An appropriately sized esophageal dilator was passed through the esophagus and into the stomach. The fundus was wrapped $270^{\circ}$ around the anterior esophagus, using interrupted nonabsorbable sutures. The fundus was sewn to both the esophageal wall and the diaphragm to maintain fixation. Although the procedure's final appearance is similar to that of Thal fundoplication, described by Aschraft et al, ${ }^{2}$ the AGF is created with an interrupted (rather than continuous) suture line. It is believed that this improves intraoperative visualization and the creation of a symmetric wrap.

The functional status of the AGF was evaluated by three approaches. (1) Each family was asked to answer standardized questions pertaining to the child's initial presenting symptoms, age, weight, and perioperative complications, as well as the current frequency of vomiting, respiratory illness, difficulty swallowing, other related symptomatology, evidence of poor growth or malnutrition, and the need for further surgical or medical management. (2) The patients were given a directed physical examination to determine their overall health and nutritional status and were admitted to the hospital for 24 hours. (3) Three clinical tests were performed to evaluate the integrity of the fundoplication, evidence of residual GER, and the possibility of delayed gastric emptying.

From the Section of Pediatric Surgery, C.S. Mott Children's Hospital and the University of Michigan Medical School, Ann Arbor, MI.

Presented at the 1993 Annual Meeting of the Section on Surgery of the American Academy of Pediatrics, Washington DC, October 29-31, 1993.

Funded and overseen by the General Clinical Research Center at the University of Michigan Medical Center.

Address reprint requests to Ronald Hirschl, MD, Division of Pediatric Surgery, C.S. Mott Children's Hospital, 1500 E Medical Center Dr, Room L2110 Women's Hospital, Ann Arbor, MI 481090245 .

Copyright 11994 by W.B. Saunders Company

0022-3468/94/2908-0024\$03.00/0 

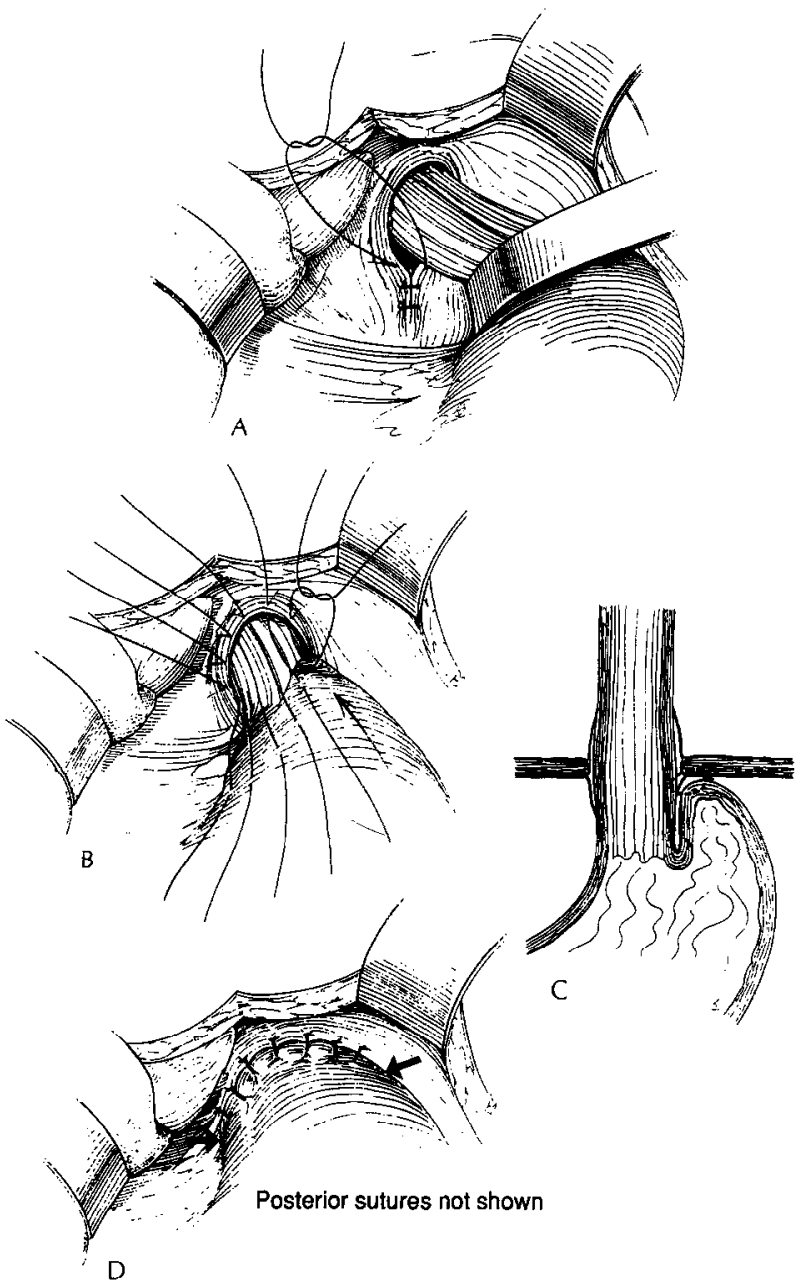

Fig 1. Illustration of the operative approach to the AGF. (A) After exposure and retraction of the esophagus, the posterior crural defect is closed in an interrupted fashion. (B) The fundus of the stomach is affixed to the esophagus and diaphragm with interrupted sutures. (C) Sutures are placed through partial thickness of each tissue. (D) The completed wrap, encircling $27^{\circ}$ (posterior sutures not shown).

The first of the clinical tests was a barium UGI, which was interpreted by one investigator (C.B.), using a previously established technique. ${ }^{3}$ All the children enrolled were able to complete this examination satisfactorily.

Twenty-four-hour $\mathrm{EpH}$, using a 4-mm probe and a portable recorder, was performed as previously described. ${ }^{1}$ Sixteen children completed this. However, in three patients, the probe was inadvertently removed at 2,10 , and 14 hours, respectively. Family members were asked to keep a diary of meals, patient position, activity, and episodes of coughing, choking, or substernal pain. Simultaneously, the $\mathrm{pH}$ monitor recorded the number of times the $\mathrm{pH}$ was below 4.0 (normal, $<50$ ), the percentage of time the $\mathrm{pH}$ was below 4.0 (normal, $<4.2 \%$ ), the number of episodes of more than 5 minutes' duration (normal, $<3$ ), and the duration of the longest episode of reflux (normal, $<9.2$ minutes). Using the formula $x+4 y$, where $x=$ the number of episodes of $\mathrm{pH}<4.0$ and $y=$ the number of episodes of $\mathrm{pH}<4.0$ for 5 minutes, a GER score was calculated. A score of more than 50 was considered diagnostic of reflux. This method has been validated previously in adults ${ }^{4}$ and has been employed in studies of infants and children. ${ }^{1}$

The third test, a liquid-phase gastric emptying study (GES), was performed to evaluate the current status of gastric motility in children treated operatively for GER. A liquid-phase study was chosen to standardize the test across a heterogeneous population, many of whom cannot tolerate solid food. These examinations were performed and interpreted by one investigator (B.S.) and were completed in 18 cases. Each child was fed a fluid meal containing technetium 99-labeled sulfur colloid (amount normalized to body weight) and was monitored with a gamma camera. The percentage of emptying at 1 hour was calculated. In our institution, normal emptying is defined as emptying of greater than $40 \%$ at 1 hour.

There were 17 males and seven females in the study group. The mean age at the time of operation was 4 years (range, 2 weeks to 22 years). Three were over age 10 , seven were 2 to 10 years of age, and 14 were age 2 or younger. Eight patients had significant neurological impairment, and two had numerous congenital anomalies. The other were healthy except for reflux-related problems. In the review of patient charts, no apparent differences were found between the study patients and those lost to follow-up, in terms of age distribution, associated neurological impairment, or perioperative complications. Statistical significance was determined by $\chi^{2}$ analysis.

\section{RESULTS}

Chart review showed no significant intraoperative complications and no perioperative deaths. Twelve patients $(50 \%)$ had minor, early postoperative complications, as illustrated in Table 1 . Two of these patients required further intervention. One underwent a secondary decompressive gastrostomy tube placement because of persistent gas bloat. The other required serial esophageal dilatations for a preexisting stricture that has since become asymptomatic. The remainder of the patients experienced no longterm sequelae of their perioperative complications.

All study patients completed the UGI and GES; 16 (73\%) finished the EpH. Of those for whom EpH data were obtained, three tolerated the examination for less than 18 hours; the remainder completed 18 to 24 hours of EpH.

Four patients (17\%) exhibited symptomatology that could be consistent with recurrent GER. The symptoms included frequent vomiting, recurrent asthma or pneumonia, and respiratory distress with feeding. However, none of the four patients believed to have GER by history had confirmation of this suspicion by objective tests. Another patient had undergone a revision of the original operation at

Table 1. Early Postoperative Complications of AGF

\begin{tabular}{ll}
\hline \multicolumn{1}{c}{ Type } & No. \\
\hline Vomiting & 4 \\
Apnea/respiratory distress & 3 \\
Prolonged ileus & 2 \\
Gas bloat & 3 \\
Esophageal stricture & 1 \\
Pneumonia & 2 \\
Abscess at gastrostomy tube site & 1 \\
\hline
\end{tabular}


another institution, 8 months after his first AGF, and remained asymptomatic.

Two groups of asymptomatic patients had evidence of GER by either UGI or EpH. Four patients (17\%) were documented to have reflux by UGI criteria. However, none of the four had symptoms, aspiration under fluoroscopy, or abnormal EpH results. Three patients $(11 \%)$ had GER documented by EpH; however, they had normal UGI results and no symptoms. All these patients remain dramatically improved from their preoperative state and are being managed nonoperatively.

Abnormal gastric emptying was an uncommon finding in this population. Three patients $(11 \%)$ had difficulty tolerating large-volume feedings and demonstrated significantly delayed gastric emptying during the liquid-phase GES. Despite this, none of these patients had symptoms or objective findings consistent with GER, nor were there growth or developmental problems.

When neurologically impaired $(\mathrm{n}=8)$ and normal children $(\mathrm{n}=16)$ were compared by objective study, there were no statistically significant differences in the incidence of recurrent symptoms or abnormal findings. One neurologically impaired child had symptoms of reflux, but this was not confirmed through further study. Another appeared to have recurrence of reflux by EpH, and a third had an abnormal GES result. Two other children appeared to have reflux by UGI. Again, no child had both symptoms and objective findings consistent with recurrent GER.

Thus, 2 years after AGF, $83 \%$ of the infants and children remain asymptomatic. Thirty-four percent have some degree of asymptomatic reflux, demonstrated by UGI or EpH.

\section{DISCUSSION}

A number of investigators have noted that recurrence of GER in infants and children is more common in the first two years after operation. ${ }^{6.7}$ Therefore, follow-up of at least 2 years was required for entry into this study. Data from this evaluation of the AGF demonstrate excellent results; $83 \%$ of the patients are clinically asymptomatic, and $66 \%$ are refluxfree by physiological testing. These clinical results compare favorably with previously published data from this institution on the efficacy of the NFP, ${ }^{1}$ as shown in Fig 2.

Ashcraft et al reported an initial success rate of $90 \%$ and a $5 \%$ reoperation rate with the $\mathrm{AGF}^{2}$ They noted that $4.5 \%$ of patients were found to have persistent symptoms suggestive of reflux, which could not be confirmed by objective study. Similarly, Caniano et al noted a reoperation rate of $6 \% .{ }^{6}$ These data,

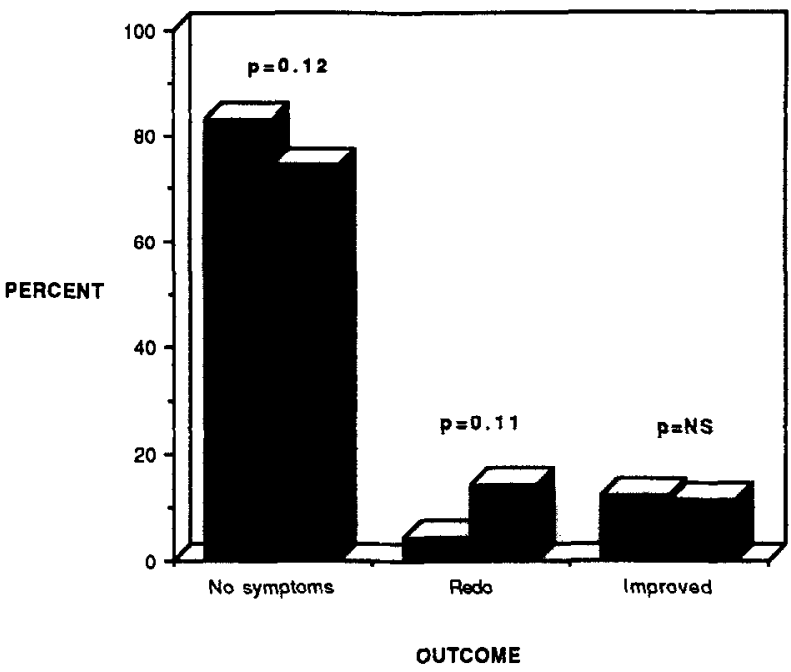

Fig 2. Comparison of outcome data for AGF (solid bars, $n=24$ ) and NFP (hatched bars, $n=35$ ). $P$ yalues are shown for each category.

combined with those obtained from this study, support both the efficacy and safety of AGF in infants and children. Moreover, although the overall complication rates are similar for NFP (45\%) and AGF $(50 \%)$, the nature and severity of these problems are disparate, as illustrated in Fig 3. Among recipients of the AGF, there were fewer perforations $(0 \% v 8.6 \%$; $P=.16)$, fewer episodes of obstruction $(0 \% v 11 \%$; $P=.16)$, fewer wound complications ( $0 \% \vee 14 \%$; $P=.15)$, and less dysphagia $(4 \% \vee 17 \% ; P=.18)$.

The finding of asymptomatic reflux, documented by either EpH or UGI, is a well-recognized, though poorly understood, phenomenon. Reported rates in follow-up studies of fundoplications vary from $3.5 \%$ to $25 \%{ }^{1,8,9}$ Although the adverse effects of peptic reflux such as stricture formation, dysplasia, and

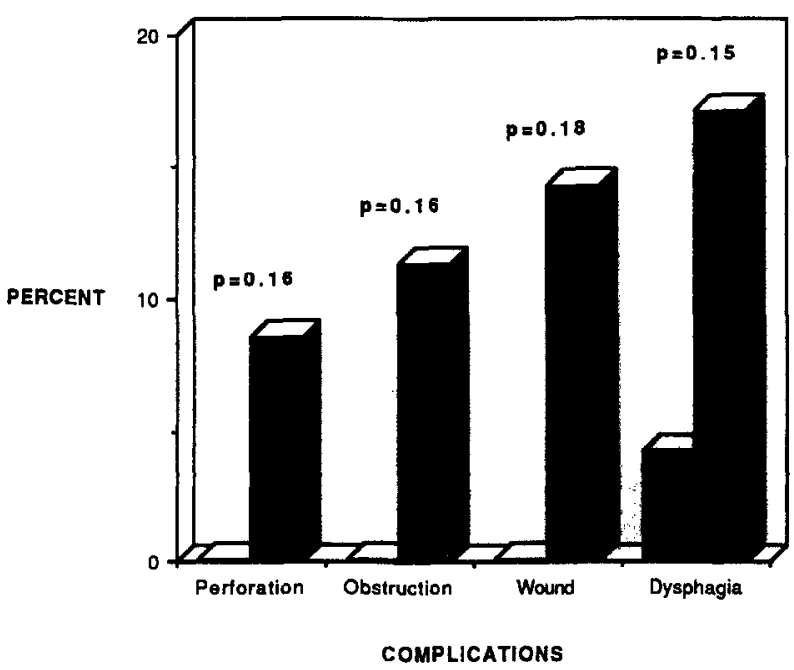

Fig 3. Complications related to AFP (solid bars, $n=24$ ) and NFP (hatched bars, $n=35$ ). $P$ values are shown for each category. 
dysmotility cannot be discounted, there are no clear guidelines for the treatment of individuals who are asymptomatic. At present, these patients are monitored closely and are treated with $\mathrm{H}_{2}$-receptor blockers and prokinetic agents.

Analysis of the experience with normal versus neurologically impaired children in this study was notable for the similarity in outcome. Concern had been raised, before completion of the study, that the latter group is at greater risk for aspiration, and therefore would fare less well if treated by any method that allowed for vomiting or eructation. However, in this population, there was no statistically significant difference in the rate of complications, symptoms, recurrence, or delayed gastric emptying between the two groups treated with AGF. This suggests that $A G F$ is appropriate for the management of GER in both neurologically normal and impaired children.

\section{REFERENCES}

1. Turnage RH, Oldham KT, Coran AG, et al: Late results of fundoplication for gastroesophageal reflux in infants and children. Surgery 105:457-464, 1989

2. Ashcraft KW, Holder TM, Amoury RA: Treatment of gastroesophageal reflux in children by Thal fundoplication. J Thorac Cardiovasc Surg 82:706-712, 1981

3. McCauley RGK, Darling DB, Leonidas JC, et al: Gastroesophageal reflux in infants and children. Am J Radiol 130:47-50, 1978

4. DeMeester TR, Wang CI, Wernly JA, et al: Technique, indications, and clinical use of 24 hour esophageal $\mathrm{pH}$ monitoring. J Thorac Cardiovasc Surg 79:656-670, 1980

5. Lefebvre F, Bouche-Pillon MA, Lefort G, et al: Surgical treatment of gastroesophageal reflux in the child using Dor's anterior partial fundoplication procedure. Report of 37 cases. Chir Pediatr 30:229-233, 1989

6. Caniano DA, Ginn-Pease ME, King DR: The failed antireflux procedure: Analysis of risk factors and morbidity. J Pediatr Surg 25:1022-1026, 1990

7. Dedinsky GK, Vane DW, Black CT, et al: Complications and reoperation after Nissen fundoplication in childhood. Am J Surg 153:177-183, 1987

8. Tunell WP, Smith EI, Carson JA: Gastroesophageal reflux in childhood. Ann Surg 197:560-565, 1983

9. Fonkalsrud EW, Ament ME, Berquist W: Surgical management of gastroesophageal reflux in childhood. Surgery 97:42-48, 1985

\section{Discussion}

C. Bagwell (Richmond, VA): This is an important subject, and with the increased emphasis on lesser procedures, we need to know if these procedures are as good as the Nissen.

I have some problems with deciding whether the anterior gastric fundoplication-or the Thal, as most of us refer to it-is as good based on the data that I've reviewed and that you've presented. First, the series is small. There were 46 patients over a 2-year period. Are these preselected patients, and if so, what criteria do you use to exclude or include them in your study.

The group of 46 patients was reduced to, effectively, 22 patients for evaluation.

About a third of the patients in the group have reflux, identified by presentation of symptoms postoperatively or by clinical testing (without symptoms). While that seems high, it's about equal to the data presented from the same institution in ' 89 and similar to the data for our own patients.

The efficacy of anterior gastric fundoplication and Nissen fundoplication appears to be about $90 \%$ long-term, although we're only looking at a 2-year period in this series. We also have to look at some of the complications.

The authors report 16 minor complications occurring in 12 patients (50\% of the patient series). The complications are not all that minor: respiratory distress episodes in three children, and gas bloat in three children. A big advantage of the anterior gastric fundoplication is that you don't get gas bloat. One of these children had it severe enough to warrant a depressing gastrostomy. Why?

About $60 \%$ of the neurologically impaired children had symptoms or objective findings of gastroesophageal reflux after this procedure. These kids have an increased risk with whatever kind of procedure we use.

We know that the Nissen procedure is good. Is the anterior gastric fundoplication less risky? And is it not as good?

R. Pearl (Washington, DC): We've been studying reflux at Walter Reed for about 6 years. We demonstrated that there is a significant difference between operations in neurologically impaired children and neurologically normal ones.

My problem with this study is, if you lump neurologically impaired and normal kids together and then report them as a group, you don't really speak to the issues. Everybody should have a high success rate in normal children; but I think we all have lots of problems with the impaired children because of the way they stress the operation. 
My question is, why did you choose to study your group the way you did? Why did you use historic controls? Why did you not just randomize your patients?

D. Bliss (response): First, we did not preselect the patients in any fashion. We simply reviewed all cases of anterior gastric fundoplication, which we began doing in 1988. We did not exclude anyone.

As to what patients we were able to include in the study, it is often very difficult to obtain follow-up on these patients, and it is particularly hard to get them involved in any invasive procedures.

We included the neurologically impaired and the nonneurologically impaired children together at the initiation of the study with the intent to, at the end, separate the data and see if there was any difference. Admittedly, with small numbers, there was no difference in the results, and so we reported on them together.

In terms of the length of follow-up, the Nissen study had a 5 -year follow-up, and this had 2 years.
However, we would agree with Dr Ashcraft's statement that $90 \%$ of recurrences of gastrocsophagcal reflux occur within 1 to 2 years' time and, therefore, this should be a relatively representative study of the recurrence of reflux, even though the follow-up period is short.

Regarding complications, there were episodes of apnea and respiratory distress, all of which were brief, short-lived, and did not require significant manipulation such as intubation.

We were very interested in why these children did have the same rate of gas bloat. We honestly don't have a good physiological answer for that question.

Two out the three children with gas bloat also had delayed gastric emptying based on the liquid-phase gastric emptying study. It may well be that these patients should undergo some form of gastric emptying study preoperatively, and if they do have delayed gastric emptying, should undergo treatment for that as well. 